\title{
Visualization of CCTV coverage in public building space using BIM technology
}

\author{
Huan-Ting Chen, Si-Wei Wu and Shang-Hsien Hsieh*
}

\begin{abstract}
Background: Nowadays, the use of Closed Circuit Television (CCTV) systems is effective for monitoring traffic, preventing crime, and ensuring safety in many public spaces. However, the effectiveness of CCTV coverage is often achieved through design experience and trial-and-error, instead of being evaluated and visualized using a robust approach.

Methods: Firstly, a method for simulating varifocal CCTV lenses in order to attain different fields of view was developed, allowing real CCTV views to be approximated by adjusting the parametric properties of simulated CCTV cameras in the 3D BIM model. Secondly, an API (Application Programming Interface) plug-in program for a commercially available BIM tool was developed to facilitate the parametric modeling of CCTV systems and the evaluation of the CCTV coverage.

Results: A complete BIM model of an MRT (Mass Rapid Transit) station was chosen as a case study to apply the developed approach to the examination of CCTV coverage. Finally, the overall coverage of the CCTV systems for the MRT station were demonstrated visually and studied in the station's BIM model.

Conclusions: This research has developed a robust visualization approach for evaluating the coverage of CCTV systems in public building spaces. The developed approach is based on Building Information Modeling (BIM) technology and is capable of simulating CCTV systems in a 3D virtual environment in order to evaluate the CCTV coverage.
\end{abstract}

Keywords: Building Information Modeling (BIM), Closed Circuit Television (CCTV) coverage, Visualization approach, Parametric modeling

\section{Background}

In the 1990s, Closed Circuit Television (CCTV) systems were used for public security and crime prevention in urban cities in England. The British government and the police were both in favor of the use of CCTV systems. On the other hand, there was a huge controversy in relation to its cost, benefits, legitimacy, and privacy issues despite its effectiveness in crime prevention (Harris et al., 1998). Nowadays, CCTV is widely used in many public spaces in order to prevent crime, monitor traffic events, and ensure public safety (e.g. Aguado et al., 2009; Teague et al., 2010).

Once a CCTV camera is installed in the field, its relocation is expensive. Therefore, a thorough design of CCTV layouts should be conducted in advance of positioning in order to ensure proper siting and mounting (Lee et al., 1995). Currently, the positions and orientations of most CCTV systems are designed based on 2D diagrams such as floor plans. This may cause many spatial design conflicts

\footnotetext{
* Correspondence: shhsieh@ntu.edu.tw

Department of Civil Engineering, National Taiwan University, Taipei, Taiwan
}

with other systems due to a lack of the third dimension in the design space, and lead to unnecessary overlapping of CCTV coverage. Additionally, both CCTV screen views and CCTV coverage are usually unknown before construction is completely finished.

Building Information Modeling (BIM) has been a rapidly-developing technology in the Architecture, Engineering and Construction (AEC) industry in recent years. Using BIM technology, an accurate building model with precise geometry and other AEC-relevant data can be constructed in a virtual environment throughout the lifecycle of the building. BIM models are thus referred to as computer-generated models that utilize parameters of model elements to support the construction, fabrication and procurement activities required to realize the building (Eastman et al., 2011: 1-2). The 3D modeling and visualization provided by the BIM software can effectively discover and solve hidden problems that previously could not be detected until construction was completed. By using different 
viewpoints, reviewers are able to conduct interference checking between systems and thus reduce unnecessary design conflicts. Also, the computational representation of a BIM model can offer a simultaneous visual representation, which allows discussion of design ideas and analytical tests during the BIM-based design process (Wang et al., 2010).

One of the major differences between BIM and conventional 3D CAD models is the linkages among model views. A conventional 3D CAD model describes a building by independent projected views such as plans, sections and elevations. If one of these views is modified, all other related views must be revised and updated manually; such a process is prone to human error. In addition, these 3D drawings contain only graphical entities, such as lines, arcs and circles. In contrast a BIM model consists of parametric objects that are defined in terms of building model elements and systems, like spaces, walls, beams and columns. BIM software is able to automatically generate conventional building plans, sections, and elevations directly from a 3D BIM model as well as photo-realistic 3D renderings, which up to a few years ago could only be produced by special visualization packages but can now be produced with little additional effort (Sah and Cory, 2008; Azhar, Hein and Sketo 2008). Furthermore, a BIM model is equipped with other non-geometric information needed for tasks in the lifecycle of the corresponding building or facility, including physical properties, functional characteristics, spatial relationships, etc. Therefore, it allows designers, engineers, and other project participants to visually examine the planning and design outcomes in a more intuitive way with richer information support. By the same token, the use of an existing BIM model to examine the coverage of CCTV systems can be a highly efficient and low cost auxiliary approach to aid CCTV layout design.

The objective of this research is to develop a robust visualization approach for evaluating the coverage of CCTV systems in public building space. The expected benefits can be summarized as follows: (1) to ensure intended CCTV coverage and reduce design conflicts with other systems; (2) to shorten the time required to setup CCTV cameras during construction; (3) to provide a better communication medium among CCTV system designers, project owners and facility operators.

In the following sections, the methods and process of modeling a CCTV system in a BIM environment are presented first. Then, an MRT (Mass Rapid Transit) station was chosen as a case study for demonstrating the results of this research. Using the Autodesk Revit Architecture (Autodesk, Inc 2010a) (hereafter shortened to Revit) along with the BIM model of an MRT station, this research employs BIM technology to integrate CCTV systems into the virtual building space of an MRT station. Finally, virtual CCTV screen views were simulated so that the CCTV coverage could be clearly visualized and studied in the virtual environment.

\section{Methods}

This section explains the process of modeling a CCTV system in a BIM model. This research uses Autodesk Revit as the development platform because the BIM model of the MRT station used in the case study was originally modeled by Revit. Revit provides a user-friendly and powerful 3D modeling design environment and can import 2D AutoCAD files for assisting with the model construction. It also provides a collaboration platform with a centralized database so that all changes can be synchronized to the cloud server (Azhar, Nadeem et al., 2008). Moreover, Autodesk supports an Application Programming Interface (API) for users to extend the core functionality of Revit through plug-in programs. In this research, a complete solution in Revit for simulating CCTV systems in BIM models is developed.

\section{Using Revit cameras as CCTV cameras}

A complete CCTV system is composed of video cameras and TV monitors. This section introduces how the Revit camera works, as well as how it can serve as a CCTV camera in order to generate virtual screen views on TV monitors. Revit supports several kinds of $3 \mathrm{D}$ views to allow users document and present projects. Among these different kinds of 3D views, the perspective 3D view can show the building model in a $3 \mathrm{D}$ view where components that are further away appear smaller, and components that are closer appear larger, as illustrated in Figure 1. Since both Revit cameras and CCTV cameras can produce perspective views on a screen, it is an appropriate and convenient option in the simulation of CCTV systems. Virtual screen views can also be automatically generated by setting up Revit cameras with several significant parameters applied.

\section{Simulating the varifocal lenses of CCTV cameras}

Different CCTV cameras in a building have different fields of view (FOV). Based on the optical properties of camera lenses, different FOVs can be achieved by changing the focal lengths of the lenses. Thus, the first step in the simulation of CCTV systems in BIM models is to come up with an approach to simulate the varifocal lenses of CCTV cameras.

Revit cameras lack parameters concerning focal length (i.e. it is not possible to change the focal length of a certain Revit camera by setting the element parameter). One possible solution is to apply a pre-calculated crop region size (see Figure 2) to the 3D view of a Revit camera, which corresponds to the desired FOV. Hence, it is necessary to figure out the relationship between the focal length (i.e. FOV) and the crop region size. According to the geometric 


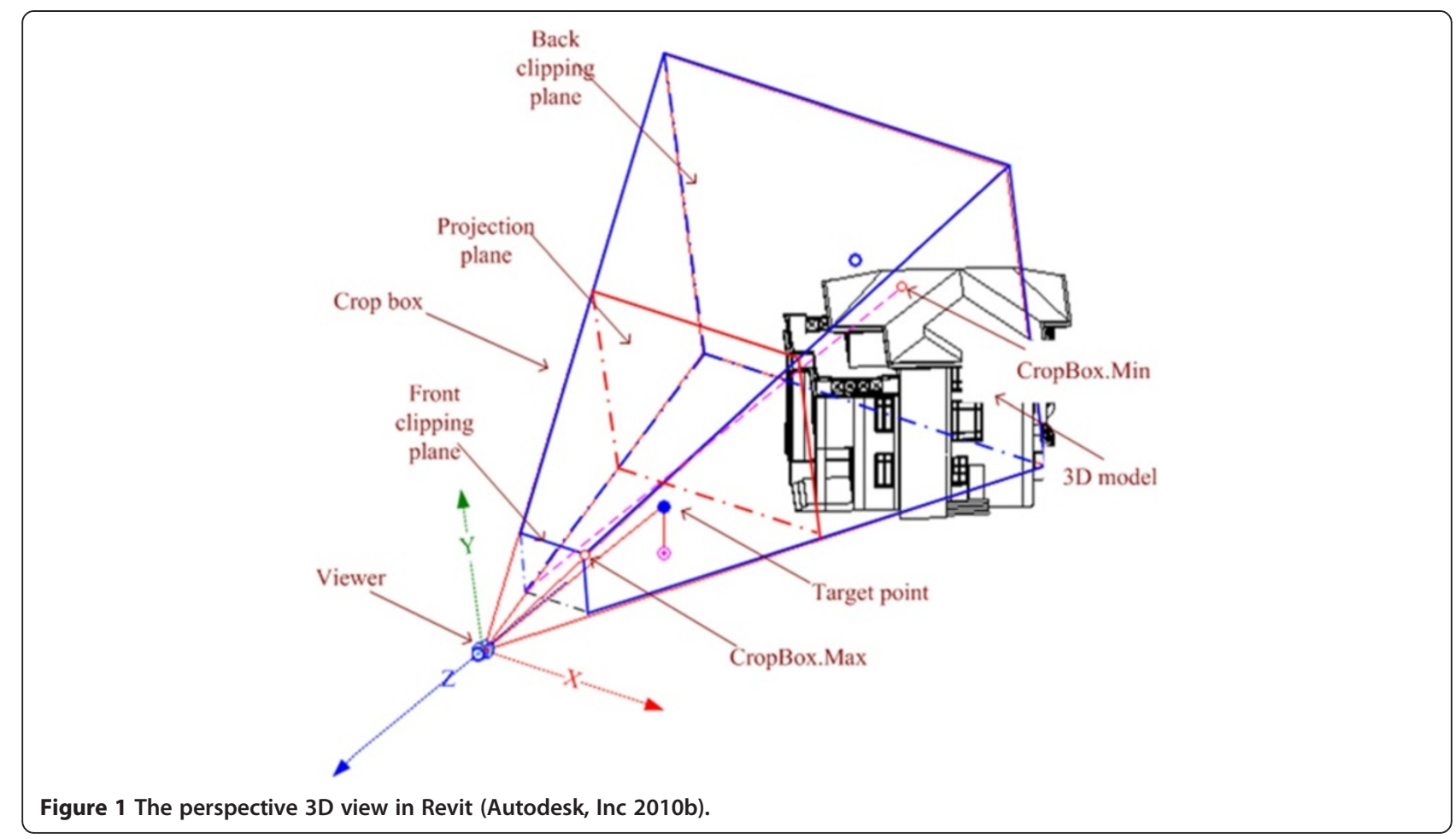

relationship, the focal length can be obtained by inputting the FOV into the following equation:

$$
\text { Focal length }=\frac{\frac{1}{2} \times \text { Film dimension }}{\tan \frac{F O V}{2}}
$$

while the default film dimension is fixed (i.e. the larger the focal length, the smaller the FOV, and vice versa, as shown in Figure 3). The width of the crop region size can now be calculated by using the principle of similar figures (see Figure 4 below). Thus, the crop region size is inversely proportional to the focal length. The height of the crop

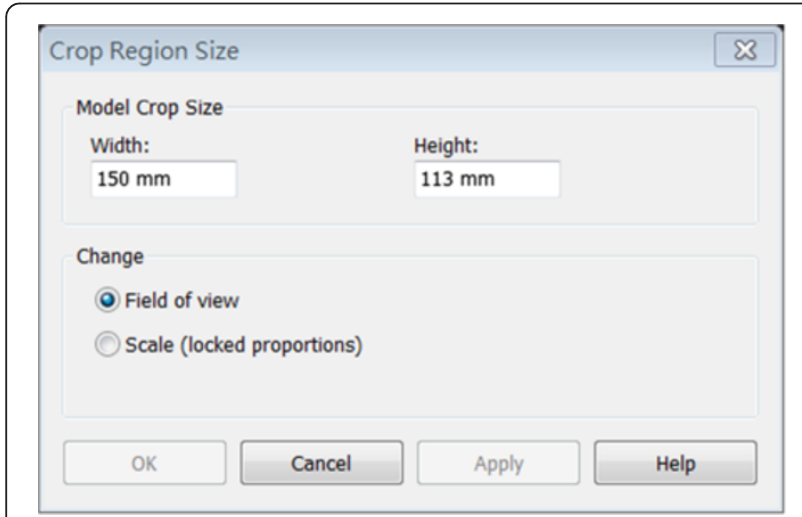

Figure $\mathbf{2}$ The menu page for setting the crop region size of the Revit camera. region can also be obtained by multiplying by the screen aspect ratio of $3 / 4$.

The default configuration of the Revit camera indicates the default field of view, the default focal length, and the default film dimension, which are 50 degrees, $38.6 \mathrm{~mm}$, and $36 \mathrm{~mm}$, respectively. In addition, the default crop region size is $150 \mathrm{~mm} * 113 \mathrm{~mm}$, as shown in Figure 2. When reconfiguration of the Revit camera for a desired FOV (for example, 60 degrees) is required, the corresponding crop region size can be calculated as follows:

(1) According to Eq. (1) and the default film dimension, $36 \mathrm{~mm}$, the focal length of 60 degrees FOV is calculated to be $31.2 \mathrm{~mm}$.

(2) According to the principle of similar figures, the width of the crop region size corresponding to 60 degrees FOV can be calculated by multiplying 150 $\mathrm{mm}$ (i.e., the width of the default crop region size) by a ratio of $(38.6 \mathrm{~mm} / 31.2 \mathrm{~mm})$, in which $38.6 \mathrm{~mm}$ is the default focal length, and the result is $185.6 \mathrm{~mm}$.

(3) According to the screen aspect ratio, the height of the crop region size can be calculated by multiplying $185.6 \mathrm{~mm}$ by $3 / 4$ and the result is $139.2 \mathrm{~mm}$.

\section{Configuring revit cameras with parameter analysis}

Virtual CCTV screen views can be simulated using Revit camera views if appropriate parameters are applied. There are several significant parameters to be configured in the CCTV system and it is necessary to relate these 




Figure 3 The larger the focal length, the smaller the FOV.

parameters to the parameters of the Revit camera. The most relevant parameters of the CCTV system to this research include FOV, camera height, target height, subject height, subject distance, and the real angle of depression. After analyzing these parameters and determining their reasonable ranges, an optimized real angle of depression $(\theta)$ for each CCTV camera can be found based on the spatial relationship shown in Figure 5. By setting up a CCTV camera with a predetermined optimized real angle of depression, construction efficiency is enhanced as the set up time is reduced. The following paragraphs explain each parameter although its reasonable range will vary from case to case. The detailed configuration data will be discussed later in the Process of modeling CCTV systems with CCTV parameter advisor section.

\section{Field of view (FOV):}

As mentioned in the previous section, a CCTV camera uses a varifocal lens to attain different FOVs, which are determined according to its requirements, location, etc. Since this research aims to examine the existing design of CCTV systems, all the FOVs of the CCTV cameras should be predefined by CCTV designers.

2. Camera height $(\mathrm{H})$ :

The camera height relates to the ceiling height. This dimension is measured from the floor elevation to the ceiling elevation. The corresponding parameter of the Revit camera is "eye elevation".

3. Target height $(\mathrm{h})$ :

Normally, the target of CCTV cameras are people, therefore, the average height of humans is adopted as the target height. The corresponding parameter of the Revit camera is "target elevation".

4. Subject height (\%) and Subject distance (D): Rotakin, developed by the Home Office in the UK, is the only device specified in BSI EN 50132-7:1996 for testing CCTV camera performance. The Home Office also offers an operation manual (Cohen et al., 2009: 8-9) that defines the concepts of subject height and subject distance. Depending on the nature of the human activity to be observed, five general observation categories have been defined for different purposes, including monitoring and control, detection, observation, recognition, and identification. These five categories are based on the relative size of a person that appears on a screen (see Figure 6). This is defined as the subject height, a percentage of the height of a person divided by the height of the screen. For a specified subject height, the maximum distance from which a person can be seen on the screen is defined as the subject distance (i.e. a person would be too small to be seen on the screen beyond the subject distance for the specified subject height). An independent consultancy company provides an online lens calculator based on the standards of the Home Office, LensCalc (CCTV Advisory Service, 2008), to help determine the subject distance required to achieve specified subject heights for different combinations of sensor size, subject height, and lens focal length (see Table 1). Please note that the subject height is used to specify

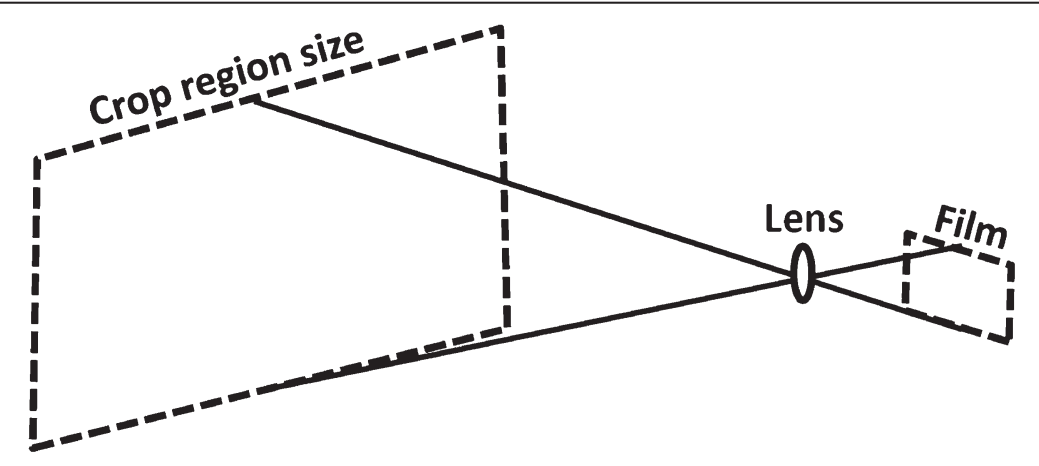

Figure 4 The principle of similar figures between the crop region size and the film dimension. 


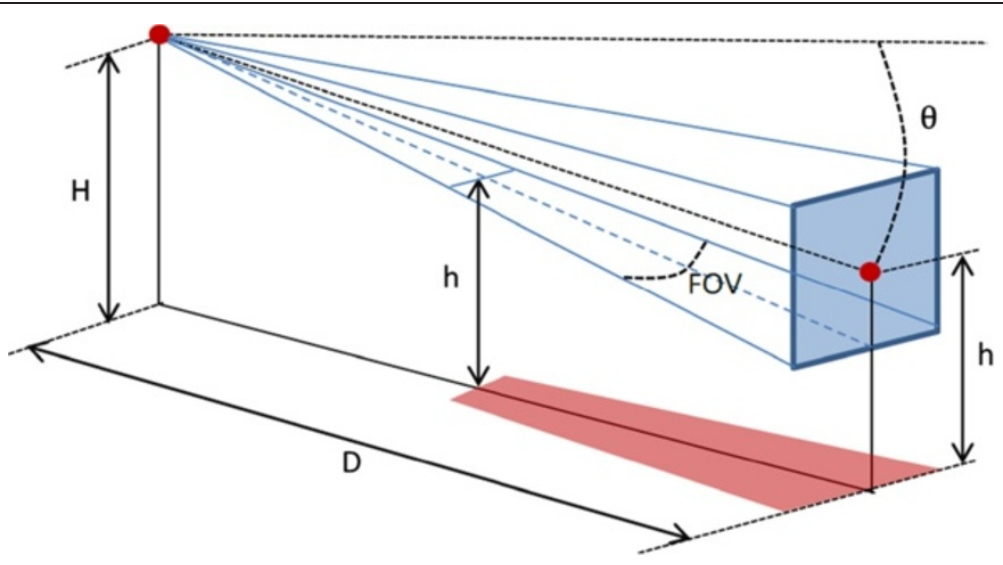

Figure 5 The spatial relationship of CCTV camera parameters.

a particular system requirement rather than to define a general standard. Additionally, the related guidelines were first developed in the PAL (Phase Alternating Line) standard, which is a color encoding standard for analogue television and has been commonly used in the design of CCTV systems in public areas. Nowadays, a smaller subject height may be accepted through the use of new cameras with higher resolutions. Please refer to the CCTV Operational requirements manual 2009 (Cohen et al., 2009: 9-10) for more information.
5. Real angle of depression $(\theta)$ :

The Real angle of depression can be referred to as the end result once the camera height, target height, and subject distance are specified. An optimized real angle of depression can be determined for each CCTV camera based on spatial relationships (see Figure 5). The following formula shows the geometric expression of an optimized real angle of depression $(\theta)$ :

$$
\tan \theta=\frac{H-h}{D}
$$
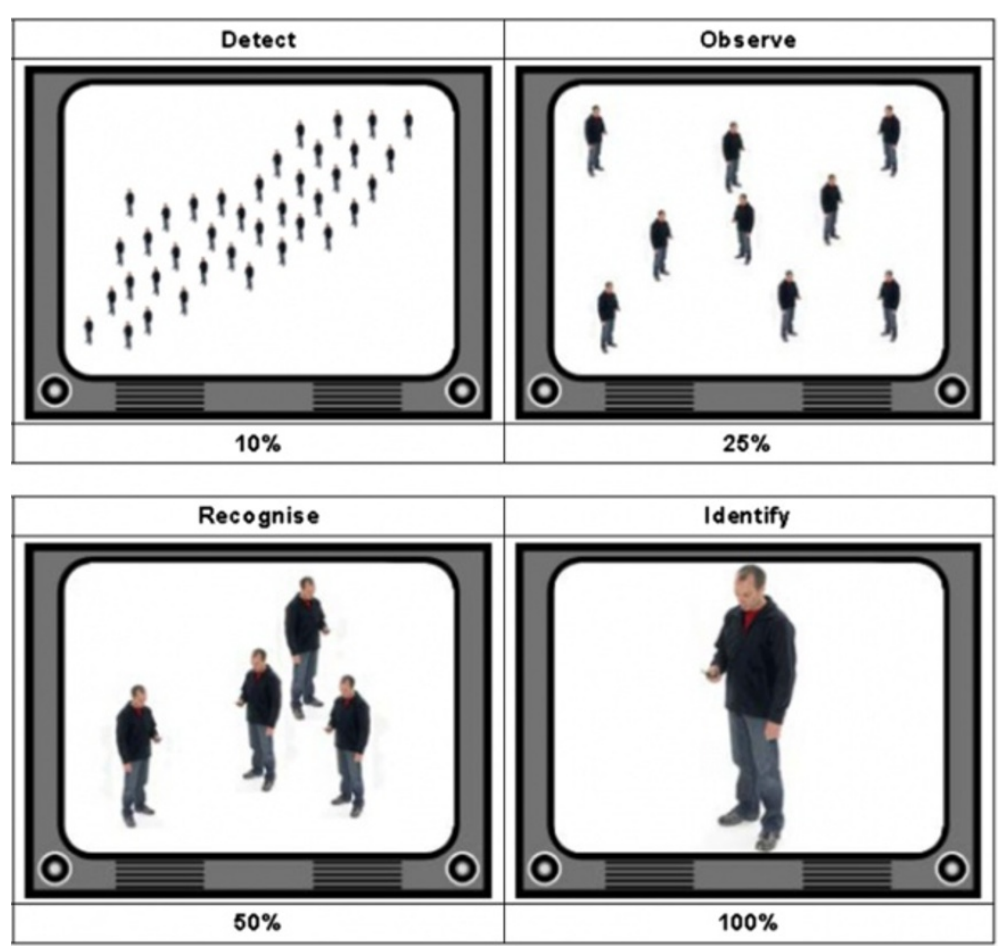

Figure 6 Four out of the five general observation categories in accordance with different subject heights (Cohen et al., 2009: 8-9). 
Table 1 Subject distances for different combinations of the subject height and the FOV

\begin{tabular}{lcccc}
\hline FOV & \multicolumn{4}{c}{ Subject height } \\
\cline { 2 - 5 } & $\mathbf{1 0 \%}$ & $\mathbf{2 5 \%}$ & $\mathbf{5 0 \%}$ & $\mathbf{1 0 0 \%}$ \\
\hline $\mathbf{1 0}^{\circ}$ & 77.72 & 38.86 & 19.43 & 9.715 \\
$\mathbf{3 5}^{\circ}$ & 21.56 & 10.78 & 5.39 & 2.695 \\
$\mathbf{5 0}^{\circ}$ & 14.6 & 7.3 & 3.65 & 1.825 \\
$\mathbf{6 0}^{\circ}$ & 11.76 & 5.88 & 2.94 & 1.47 \\
$\mathbf{9 5}^{\circ}$ & 6.24 & 3.12 & 1.56 & 0.78 \\
$\mathbf{9 9}^{\circ}$ & 5.8 & 2.9 & 1.45 & 0.725 \\
\hline (Unit: meter). & & & &
\end{tabular}

In addition to the parameters above, the CCTV coverage is also shown in Figure 5. The red trapezoidal area reveals the coverage area of a certain CCTV camera that covers the area where persons standing can be seen by the CCTV for a specified subject height. The measure of that red trapezoidal area can also be calculated and displayed in BIM models using Revit as discussed later in the Evaluation and visual representation of CCTV coverage section.

\section{Simulating virtual CCTV screen views}

As previously mentioned in the Using Revit cameras as CCTV cameras section, a Revit camera object that provides a $3 \mathrm{D}$ perspective view can serve as the CCTV camera in the Revit BIM environment. That is, virtual CCTV screen views can be simulated by using Revit camera views if appropriate parameters are applied.

In order to evaluate the existing design of CCTV systems, it is essential to accurately position all CCTV cameras within the BIM model according to the plan views of the CCTV design in 2D CAD files. After importing the 2D CAD files of CCTV plan views and aligning them with the floor plan views of the BIM model (see Figure 7), Revit cameras can be properly positioned with reference to the layout of the two overlapped plan views. Virtual CCTV screen views can also be automatically generated after inputting all of the required parameters (see Figure 8), including eye elevation, target elevation, etc. Figure 9 shows an example of a virtual CCTV screen view simulated by the Revit camera. It is important to carefully determine the values for the parameters of Revit cameras based on those of their corresponding CCTV systems.

Because the MRT station used in the case study is still under construction, the capture of a real view image from a CCTV camera in the station for a validation study is not possible. However, to validate the proposed approach for simulating the CCTV cameras by the Revit cameras, a comparison was made between a real photo






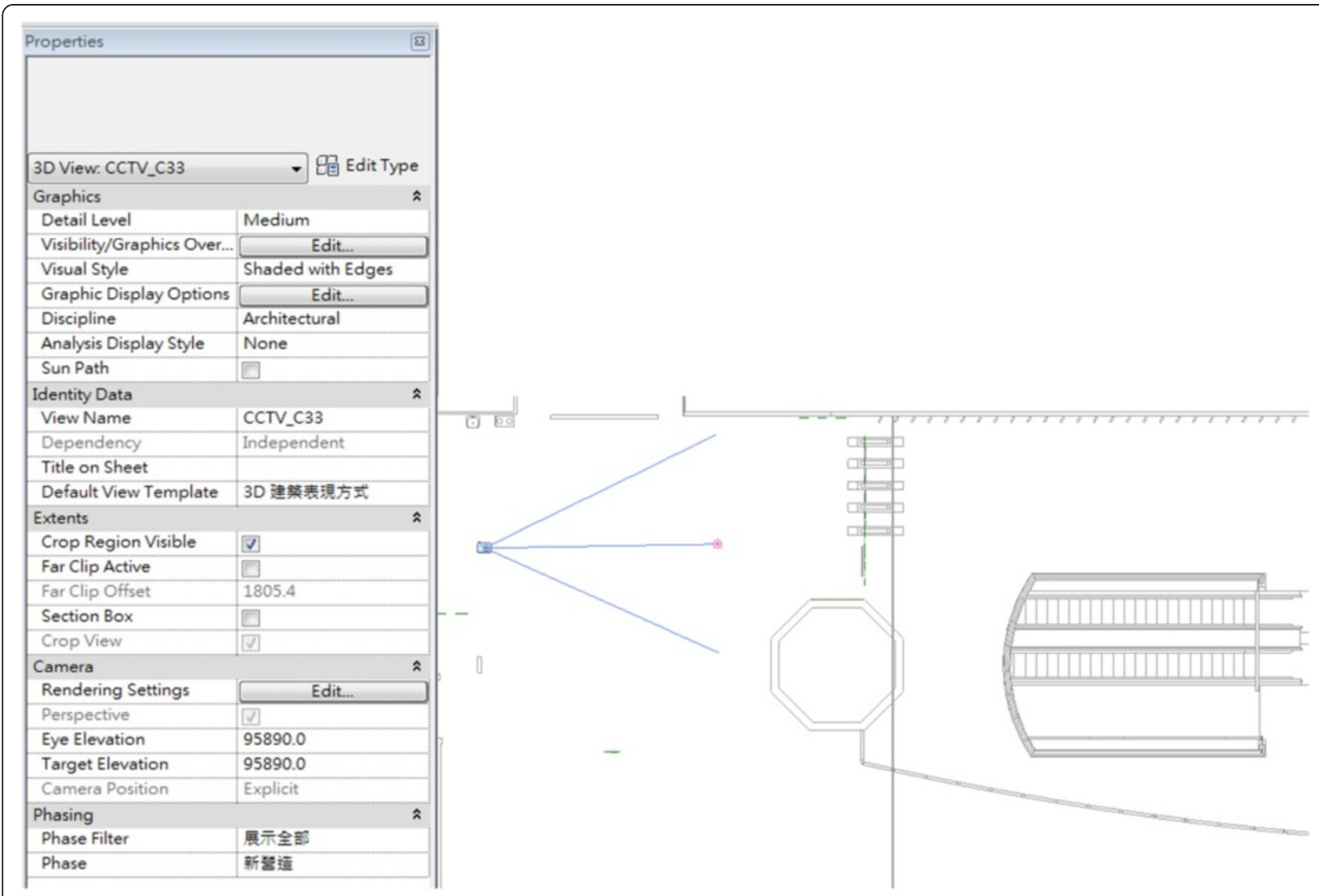

Figure 8 Configuring the parameters of the Revit camera.

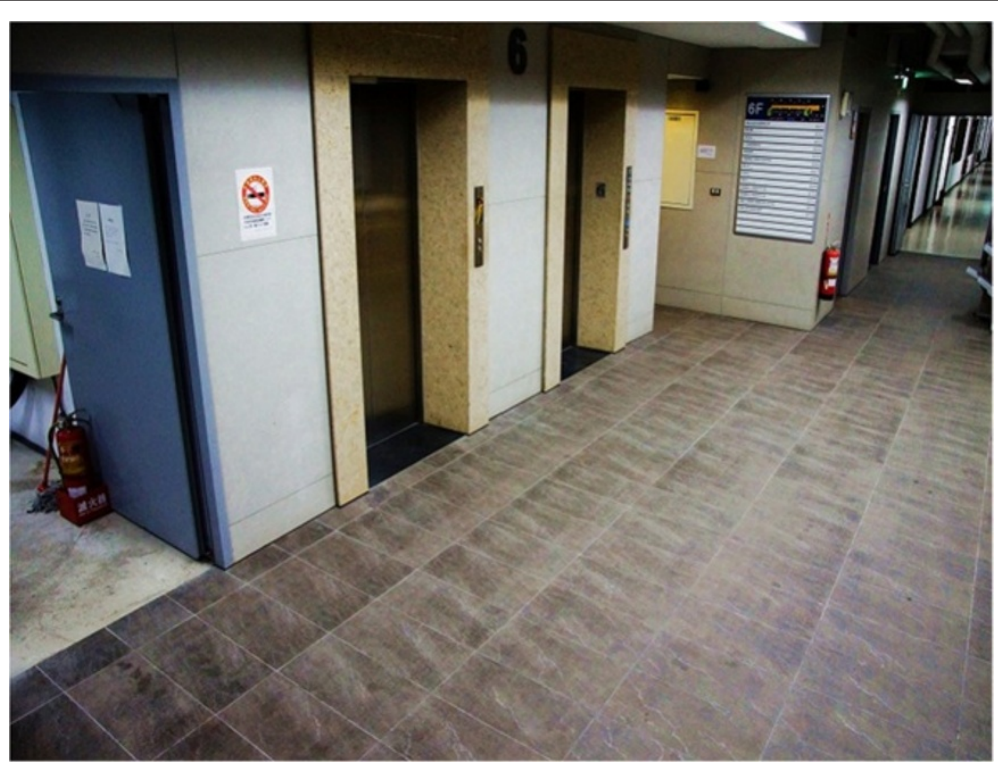

Figure 9 A real photo taken with the digital camera (SONY SLT-A77V). 


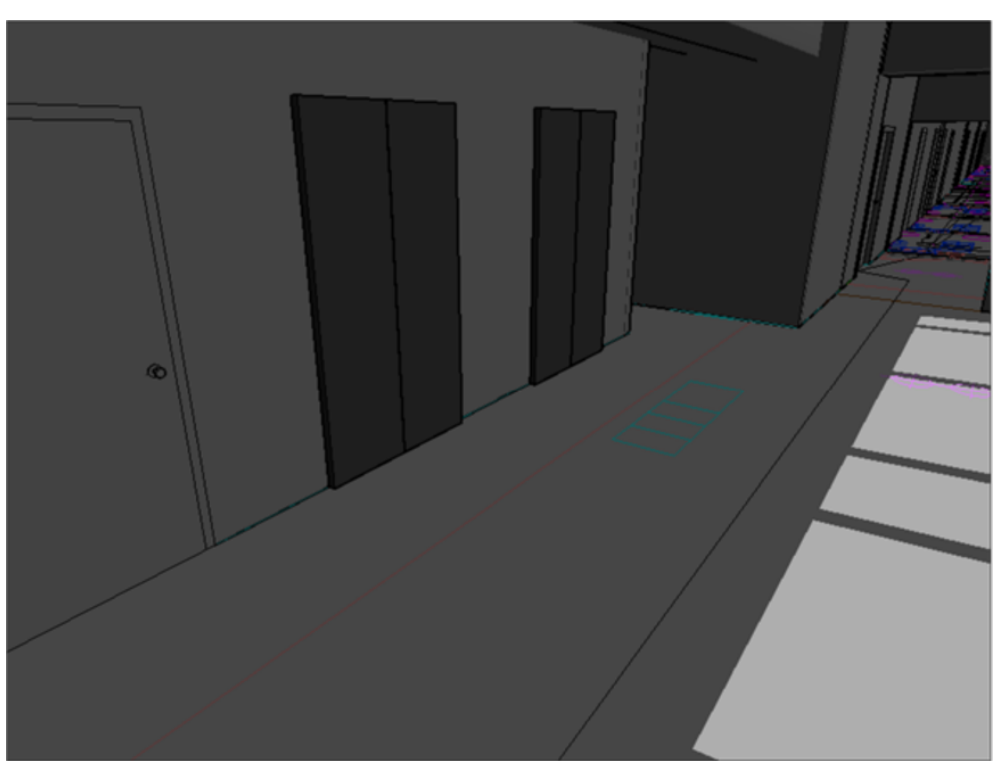

Figure $10 \mathrm{~A}$ virtual CCTV screen view simulated by the Revit camera for comparison with the photo in Figure 9.



Figure 11 The user interface of the CCTV setup advisor for Revit cameras. 


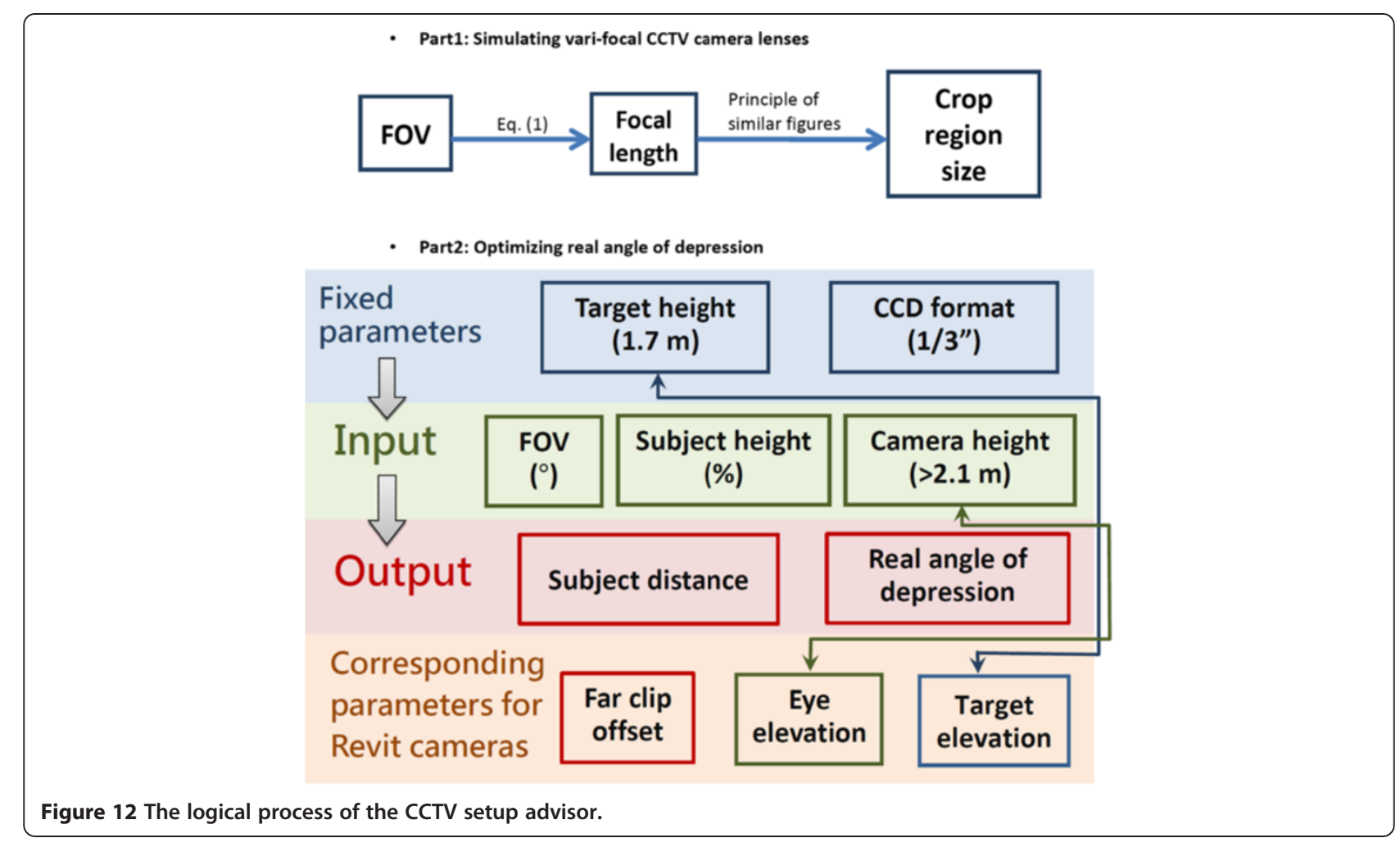

taken by a digital camera (SONY SLT-A77V) and an image simulated by a Revit camera at the same location on the $6^{\text {th }}$ floor of the Civil Engineering Research building at the National Taiwan University (see Figures 10 and 9 , respectively). It can be seen that a satisfactory simulation result is obtained.

\section{Plug-in development with revit API}

Autodesk has released the Revit API (Autodesk, Inc $2010 \mathrm{~b})$ to allow users to develop plug-in programs in order to automate repetitive tasks, thus extending the core functionality of Revit. With Revit API, it is also possible to perform customizations based on the

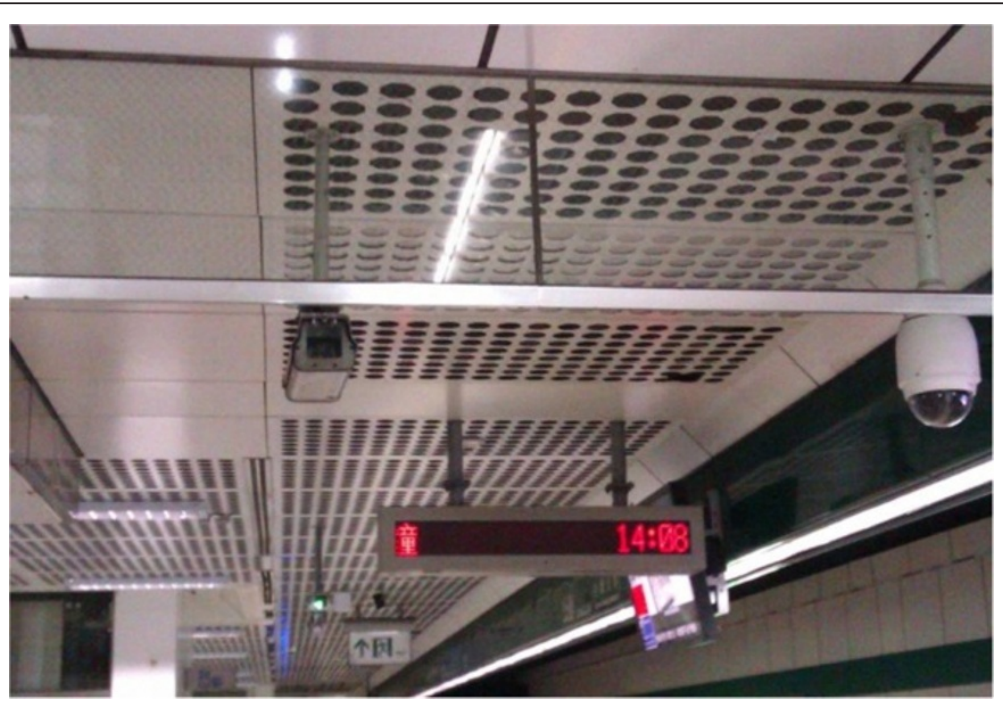

Figure 13 The heights of CCTV cameras are lower than normal due to the influence of the smoke curtain. 




Figure 14 Smoke curtains on the concourse level (CCTV No. C29, the location is shown in Figure 24).

requirements of users. The program developed with Revit API is called Revit add-in that supports all .NET compliant languages for programming.

This research uses Autodesk Revit Architecture 2010 as the BIM software. It also provides an API to enable advanced users to develop external applications in order to incorporate BIM-based parametric design methods (Wang et al., 2010). The API offers access to the active Revit document and its corresponding BIM database. Information can be retrieved from the database, and any External Commands available in the Revit API can perform basic database operations (Yan et al., 2011).

To enhance the performance of Revit in the evaluation of CCTV coverage, a plug-in API program, called CCTV setup advisor for Revit cameras (hereafter shortened to CCTV setup advisor) is developed in this research with the following two considerations. Firstly, it is more succinct to integrate all related functions and steps in Revit to a single interface because modeling CCTV systems in BIM models requires a sequence of procedures. Secondly, it is more time efficient for users to configure many parameters in a single interface due to the fact

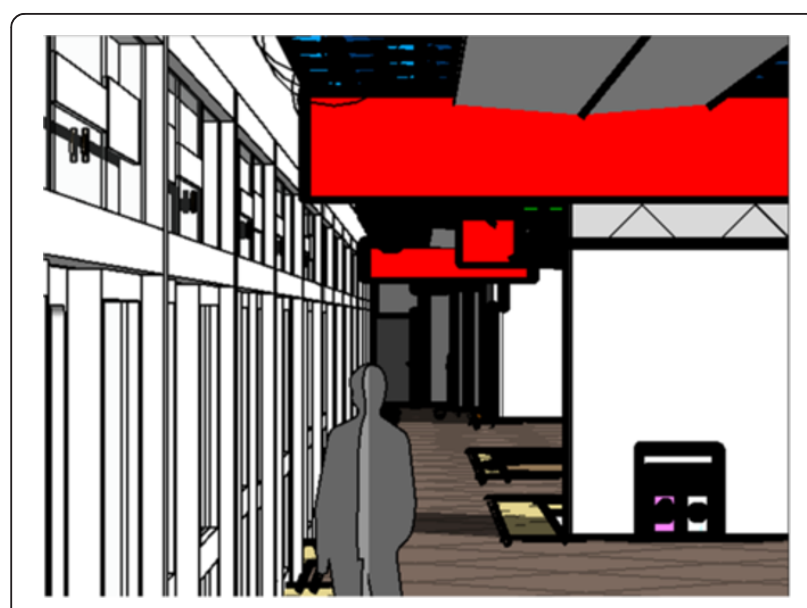

Figure 15 Ceiling signboards on the platform level. (CCTV No. $\mathrm{C} 2$, the location is shown in Figure 25).

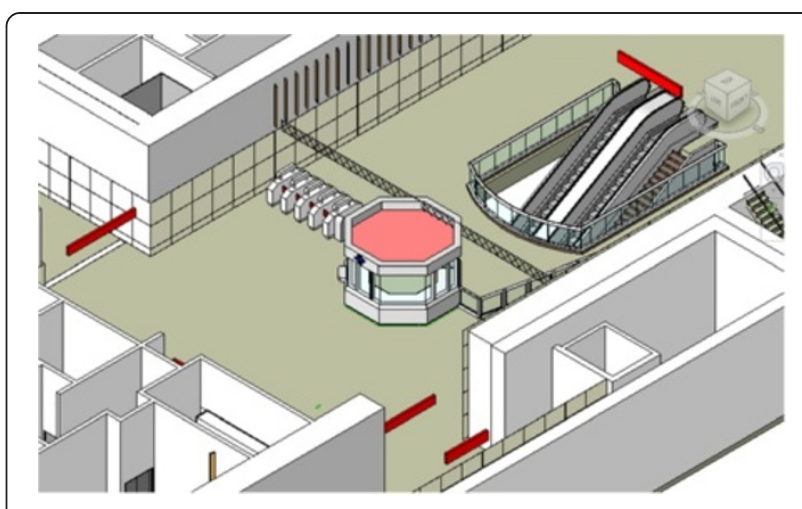

Figure 16 A bird's eye view of smoke curtains and ceiling signboards on the concourse level.

that there may be many CCTV cameras required even in a single building space.

CCTV setup advisor, therefore, has two main functions developed with BIM technology: (1) simulating varifocal CCTV camera lenses by Revit cameras and (2) configuring the parameters of Revit cameras to obtain virtual CCTV screen views and optimized real angles of depression. Revit cameras in the Revit API are read-only; therefore, no parameter of Revit cameras can be modified through external API commands. Thus, it is advisable for users to manually input the values of the parameters suggested by the plug-in program.

Figure 11 shows the user interface of the plug-in program. The upper section simulates varifocal CCTV camera lenses, which helps to determine the crop region size depending on different FOVs. The lower section optimizes the real angle of depression, which suggests parameters for Revit cameras based on user inputs and then calculates the real angle of depression. Figure 12 presents the logical process of this plug-in program, including input of parameters, returned outputs, and the relationship between parameters. An additional movie file is provided to show the CCTV setup advisor in more detail (see Additional file 1).

\section{Results and discussion}

After successfully modeling a CCTV system in a BIMbased virtual building space, this research conducted a case study to examine CCTV coverage. To make the BIM-based virtual environment correspond better to a real situation, a BIM design model of an MRT station under construction in Taipei city was adopted for the case study.

The application of CCTV systems in public spaces, especially mass transit facilities, for surveillance and protection purposes has grown at a considerable rate (Sanderson et al., 2007; March Networks News, 2008). Some research, focused on long-term experiments of advanced Intelligent CCTV (ICCTV) technologies in some 


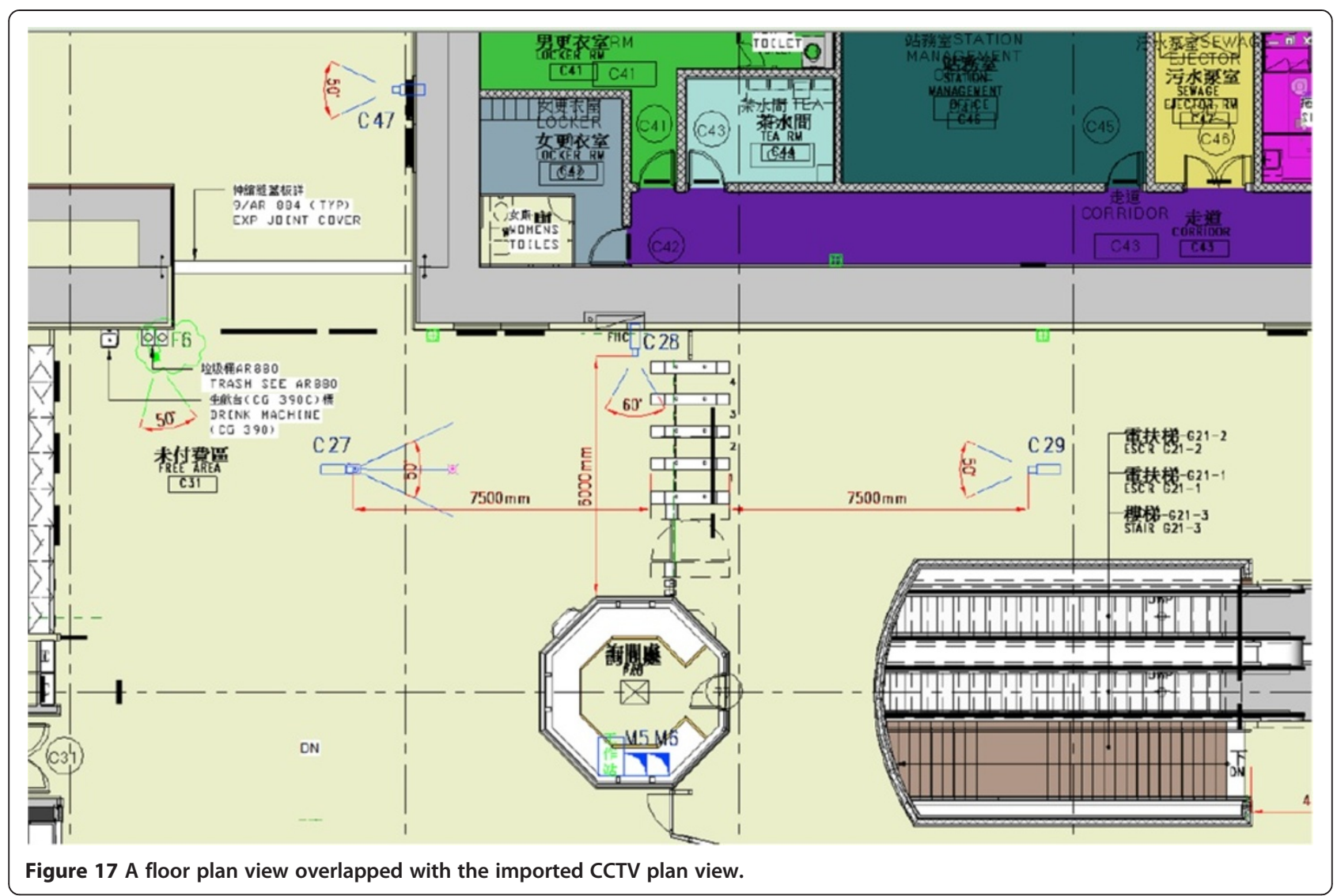

sensitive public spaces, like major ports and railway stations, has also been published (Bigdeli et al., 2007). In Taipei city's MRT system, the CCTV systems are mainly used at platforms and in the lobbies of an MRT station for the following functions (Sun, 2005):

1. When the MRT train stops at a station, the train driver can monitor the movement of passengers getting on and off the train via CCTV systems. This helps to ensure the safety of passengers.

2. Station staff can monitor the activities in the station through the CCTV systems so that proper reactions can be made promptly in the case of an emergency.

3. The traffic control center manages the operations of the entire MRT system with the help of the CCTV systems in MRT stations. The operators can visualize all situations in MRT stations and respond correctly to ensure the safety of the trains in service.



Figure 18 Crop region size advised by the CCTV setup advisor.
4. Station staff can monitor, through CCTV systems, the traffic flows of passengers at all entrances and exits of the station and ensure that entry/egress is smooth.

The purposes of application discussed above also define the design requirements of the CCTV systems in the MRT system. For example, CCTV cameras are needed in certain locations on the platform to ensure full coverage of the movement of passengers getting on and off the trains. They are also needed at all entrances and exits of stations for good coverage of passenger traffic flows.

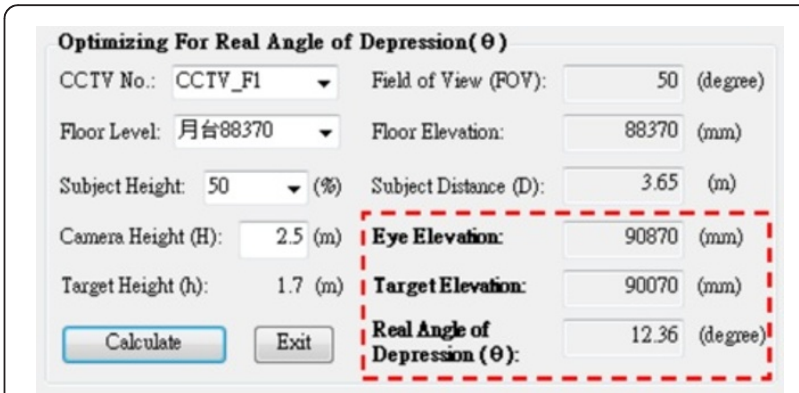

Figure 19 Parameters suggested by the CCTV setup advisor. 


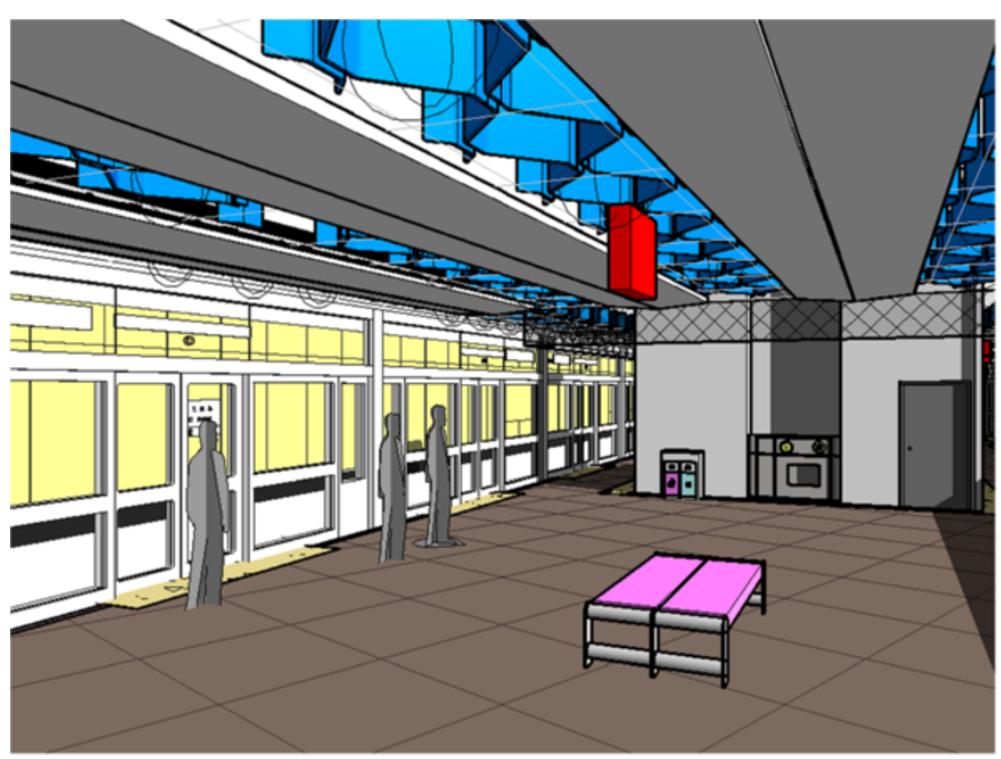

Figure 20 A virtual CCTV screen view simulated by the Revit camera (CCTV No. C18, the location is shown in Figure 25).

Modeling of smoke curtains and ceiling signboards In order to examine any design conflicts between CCTV systems and other systems, several architectural components in an MRT station that may block the views of the CCTV cameras need to be modeled as parametric elements in the BIM model. These include smoke curtains and ceiling signboards.

Traditionally, the placement of a CCTV camera is subject to the conditions of the on-site environment, such as irregular ceiling heights, suspended signboards under the ceiling, smoke curtains, and so on. For example, in the real MRT station shown in Figure 13, the CCTV cameras are located lower than the normal height due to the influence of the smoke curtain. A lower location for a CCTV camera can increase the possibility of being intentionally destroyed and decrease the range of the CCTV coverage. If a better location can be found in advance using the BIM model, a suitable height for the CCTV camera can also be determined. However, the consideration of these architectural components has relatively low priority during the life cycle of the construction project and may not be modeled in the BIM model. This is also the case for the BIM model the authors obtained from the design company.



Figure 21 A simulated CCTV screen view before the checking of design conflicts. 


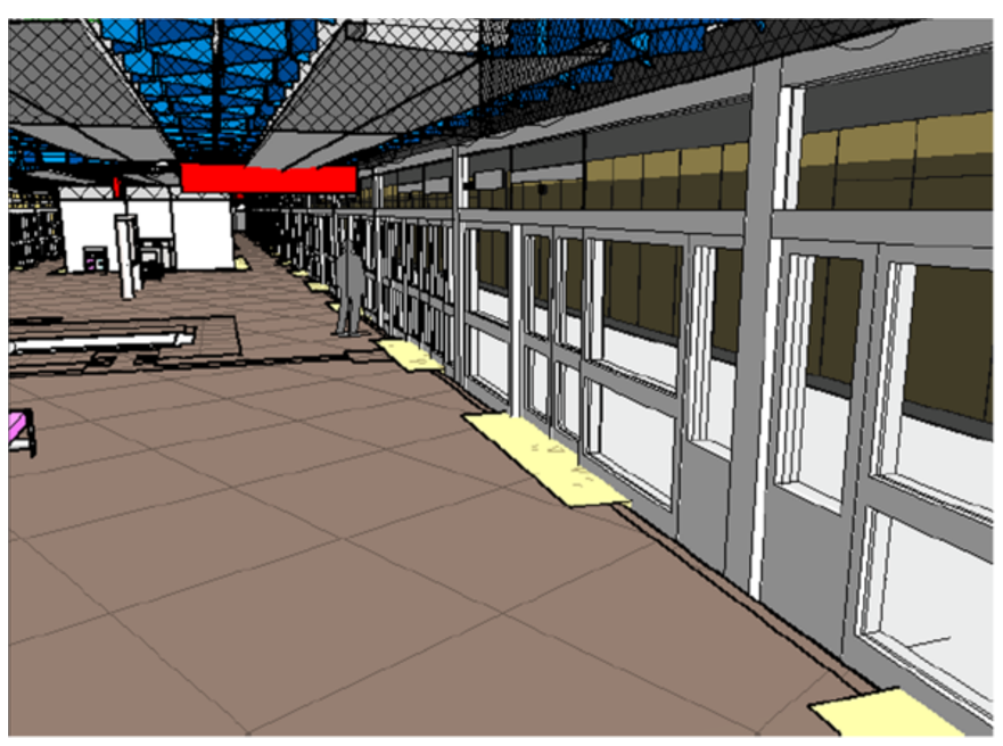

Figure 22 A simulated CCTV screen view after the checking of design conflicts (CCTV No. C10, the location is shown in Figure 25).

Thus, this research models the smoke curtains and ceiling signboards as parametric elements in the Revit model. Among predefined system family types in Revit, the Wall element is most appropriate for modeling parametric smoke curtains and ceiling signboards. By means of modifying several Wall properties, such as base constraint, base offset, top constraint, top offset, and thickness, the appearance of Wall elements can resemble smoke curtains and ceiling signboards. The elements with different locations, heights and sizes can be easily and parametrically modeled in the BIM model.

In order to distinguish smoke curtains from ceiling signboards, the two kinds of elements were assigned different materials, colors, and appearances. Smoke curtains are set to be transparent meshed acrylic sheets, as shown in Figure 14, while ceiling signboards are set to be red opaque acrylic sheets, as shown in Figure 15. In addition, Figure 16 presents a bird's eye view of both smoke curtains and ceiling signboards on the concourse level of the MRT station.

\section{Process of modeling CCTV systems with CCTV parameter advisor}

The first step of modeling CCTV systems is to correctly position Revit cameras according to the existing design drawings. Thanks to the assistance from the Department of Rapid Transit Systems, Taipei city government, 2D plan views of the CCTV system in the MRT station were provided in CAD format and therefore could be imported into the BIM model directly. With reference to the imported CCTV plan view, Revit

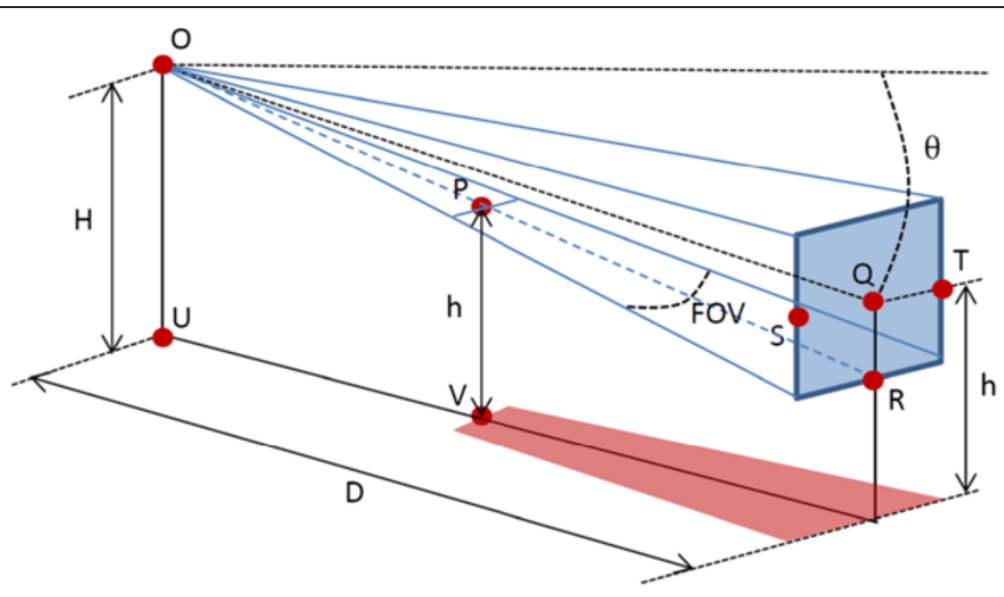

Figure 23 The calculation of the trapezoidal coverage area. 


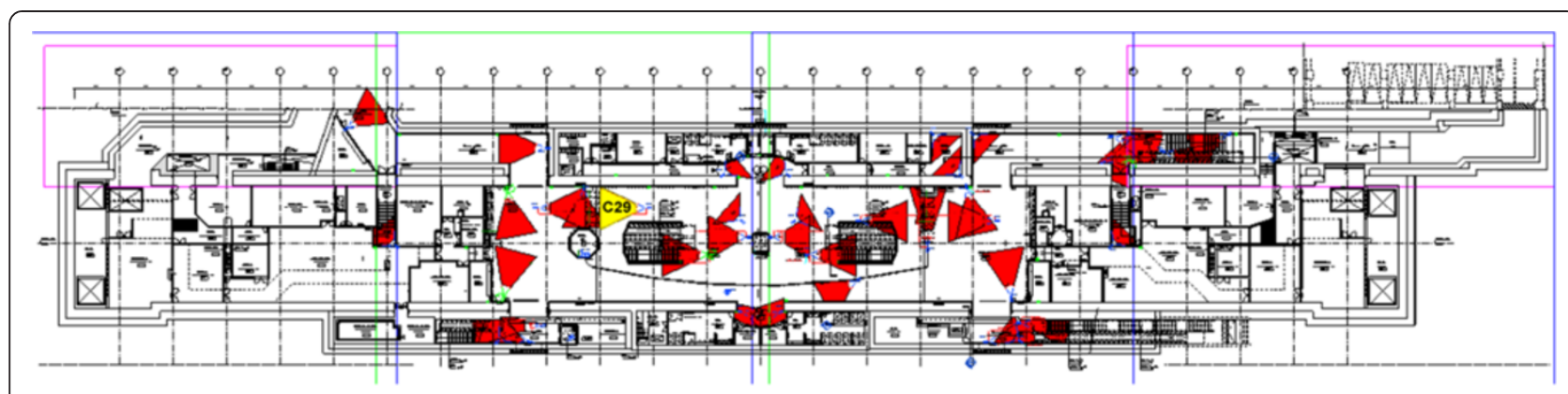

Figure 24 The CCTV coverage of the concourse level shown in a 2D floor plan view (Coverage ratio $=25.73 \%$ ).

cameras can be placed in accurate positions on the corresponding floor plan view of the BIM model (see Figure 17). For modeling CCTV cameras with different FOVs, the developed plug-in program, CCTV setup advisor, can be used for calculating the corresponding crop region size (see Figure 18).

The second step is to determine the other two necessary parameters for the Revit cameras within reasonable ranges: the eye elevation and the target elevation. As previously mentioned in the Configuring Revit cameras with parameter analysis section, the eye elevation depends on the camera height, while the target elevation depends on the target height. Normally, the reasonable range of the camera height may be between 2.3 and 2.5 meters in the environment of an MRT station. Also, it is appropriate to apply the average height of the Taiwanese people to the target elevation, that is, approximately 1.7 meters.

After creating all necessary Revit cameras in the first step, the CCTV setup advisor is able to read in all Revit camera instances as well as the elevations of all floor levels, as shown in Figure 19. Subsequently, users need to key in the correct eye elevation and target elevation values for the Revit cameras (see Figure 8), which can be advised by the CCTV setup advisor after the selection of the desired Revit camera, the floor level, and the subject height, and the input of desired camera height. As for determining the subject height, it should be chosen based on the monitoring requirements of users (see the discussions in the Configuring Revit cameras with parameter analysis section and Figure 6). It should be noted that the selection of the subject height does not affect the simulation of the CCTV view but the effective coverage of the simulated CCTV. As shown in Figure 20, a CCTV screen view can be simulated by the corresponding Revit camera view. The simulated views are accessible when users choose a certain Revit camera view from the list of all 3D views via the Revit project browser.

More importantly, the CCTV setup advisor can suggest an optimized real angle of depression for the specified CCTV camera (see Figure 19). This may help to reduce the time for installation and adjustment of CCTVs because the best angle of depression for a CCTV camera has been determined in advance under userdesired conditions (i.e. an optimized real angle of depression exactly corresponds to a virtual CCTV screen view simulated by the Revit camera).

\section{Checking for design conflicts}

Prior to the use of 3D technology, detecting interferences between systems was both difficult and time-consuming. Nowadays, 3D BIM technology helps detect clashes in advance through more sensible visual presentations and

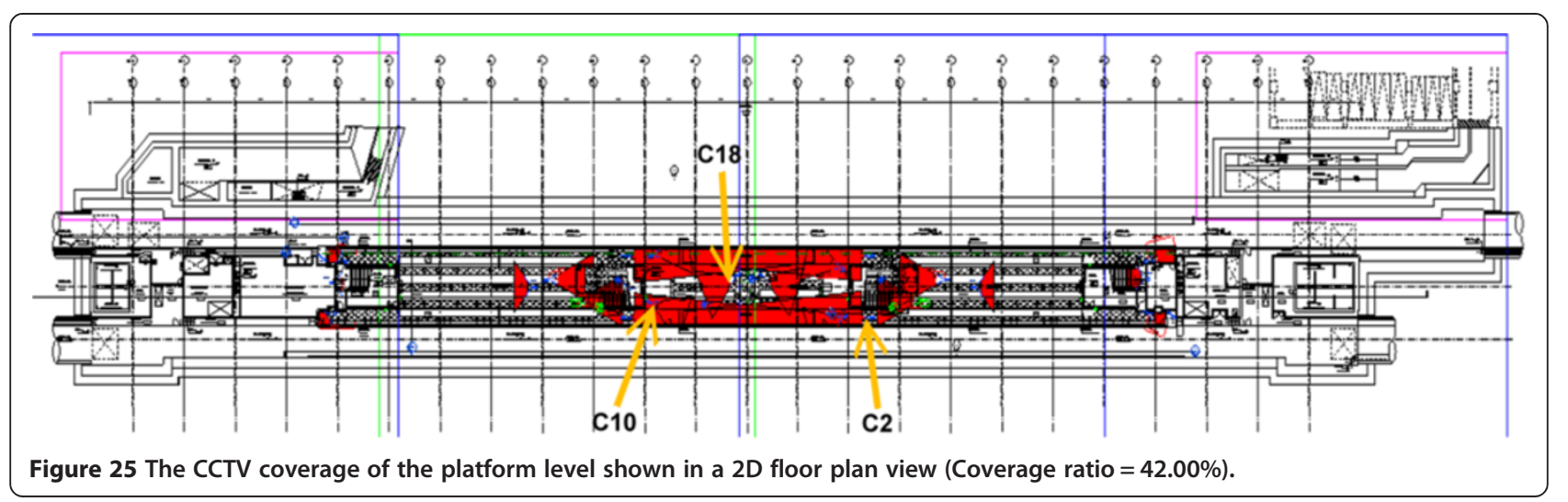






Figure 26 The CCTV coverage of the concourse level shown in a 3D model view (Coverage ratio $=25.73 \%$ ).

more seamless collaboration platforms (Eastman et al., 2011: 272-273).

One of the aims of this research is to take advantage of BIM technology for inspecting in the design phase whether a desired location for CCTV camera installation is suitable in the future construction phase. Once CCTV screen views can be simulated via Revit cameras, it is simple to check if anything blocks the view of the CCTV camera in the BIM model. Examples of these are smoke curtains and ceiling signboards, which can be modeled as parametric elements in the BIM model of the MRT station. Such design conflicts must be discovered in advance and eliminated to reduce the installation and adjustment time required for the setup of CCTV cameras.

However, it is difficult to give a precise definition of design conflicts due to the fact that how seriously something blocks the CCTV camera's view may depend on subjective assessment by the operators. A more objective way to evaluate whether there is a clash is to use the optimized real angle of depression suggested by the CCTV setup advisor. Once an optimized real angle of depression is applied, most interference is supposed to be avoided. If there is still an obstacle blocking the CCTV camera's view, this implies that the parameters of the CCTV camera should be reset, including the camera height, target height, FOV, or even its original location.

Figure 21 shows a simulated CCTV screen view where almost half of the view is blocked by smoke curtains and ceilings. After adjusting several parameters of the Revit camera with the assistance of the CCTV setup advisor, a clearer view can be obtained as shown in Figure 22. The setup of a CCTV camera must be based on the

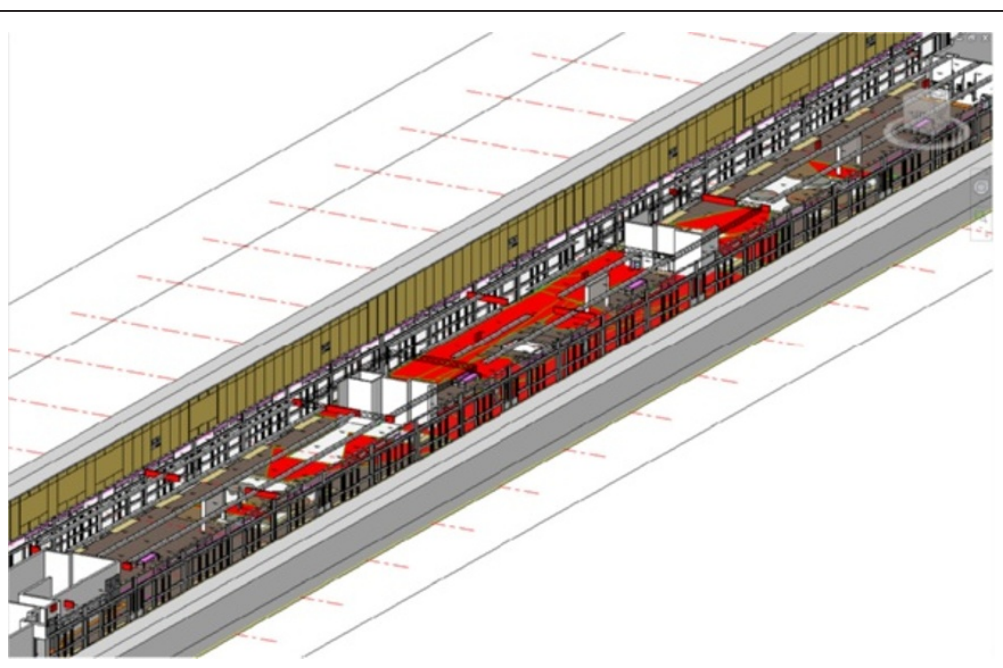

Figure 27 The CCTV coverage of the platform level shown in a 3D model view (Coverage ratio $=42.00 \%$ ). 
monitoring requirements of users. The simulation of CCTV screen views offers an approach for CCTV designers to conduct both detailed modifications and subtle adjustments prior to real construction.

\section{Evaluation and visual representation of CCTV coverage}

Even with the highly developed VR technology used today in the construction industry, CCTV systems are more often designed in a $2 \mathrm{D}$ environment. As CCTV systems have been introduced in 3D BIM models in this research, it is convenient to display the overall 3D environment and the CCTV coverage area through both 2D and 3D VR approaches.

In order to display visual representations of CCTV coverage, it is essential to define the effective area covered by CCTV cameras. According to Figure 23, the red trapezoidal area reveals the coverage area of a certain CCTV camera. For a known FOV, the area can be determined by the subject distance (D) and the length $\overline{U V}$. Because the subject distance (D) and other parameters, such as $h, H$, and FOV, have been defined in the Configuring Revit cameras with parameter analysis section, the length of $\overline{U V}$ can be calculated using the following steps:

(1) Assuming that the coordinates of the point $\mathrm{O}$ and the point $\mathrm{Q}$ are $(0,0, H)$ and $(\mathrm{D}, 0, \mathrm{~h})$, respectively.

(2) The length of $\overline{O Q}=\sqrt{(\mathrm{H}-\mathrm{h})^{2}+\mathrm{D}^{2}}$.

(3) The length of $\overline{S T}=\overline{2 O Q} \times \tan \frac{F O V}{2}$, where the point $\mathrm{Q}$ is the midpoint of the line segment, $\overline{S T}$.

(4) Based on the screen aspect ratio (i.e. 3/4) as mentioned in Simulating varifocal lenses of $C C T V$ cameras section, the coordinate of the point $\mathrm{R}$ is $\left(\mathrm{D}, 0, \mathrm{~h}-\frac{1}{2} \times \frac{3}{4} \overline{S T}\right)$.

(5) The coordinate of the point $\mathrm{P}$ is $(\overline{U V}, O, h)$ and the points of $\mathrm{O}, \mathrm{P}$ and $\mathrm{R}$ are in the same straight line. Therefore, the length $\overline{U V}=D(H-h) /\left(H=h+\frac{3}{8} \overline{S T}\right)$.

Although the red trapezoidal area can be calculated through the mathematical approach, it is more convenient and efficient to use the "Filled region" function in Revit. Once an area formed by a closed loop of lines is specified, the corresponding filled region can be shown on the floor plan view, and the measure of that area can be calculated automatically.

After all "filled regions" are created for all CCTV cameras in the MRT station, it is crucial to check if there are any overlapping areas among those filled regions. If so, the filled regions with overlapping areas must be redrawn as a single closed loop area to obtain accurate calculations of the areas. Although this may take a lot of time to do, especially when there are many CCTV cameras in an MRT station, it is a necessary step.
Finally, the coverage ratio can be determined by dividing the total area of all filled regions by the overall floor area in the MRT station. The overall effective coverage of the CCTV cameras in the MRT station can be displayed on both 2D floor plan views and 3D model views. Figure 24 and Figure 25 show the CCTV coverage in 2D floor plan views of the concourse level and the platform level, respectively. Figure 26 and Figure 27 show the effective CCTV coverage in 3D model views of the concourse level and the platform level, respectively. An additional movie file is provided to show the CCTV coverage in the MRT station in more detail (see Additional file 2).

\section{Conclusions}

This research has developed a robust visualization approach for evaluating the coverage of CCTV systems in public building spaces. Firstly, a method for modeling CCTV systems in virtual building spaces is presented. The emphasis is placed on offering a visual representation of the CCTV coverage in a BIM-based virtual environment. By simulating varifocal lenses and configuring the parameters of Revit cameras, the developed approach simulates the CCTV screen views to provide a better visual demonstration of the working of the CCTV systems. This is advantageous in the checking of design conflicts and effective communication between owners and contractors. The filled regions displayed in the 3D environment are also apparent, allowing accurate visual evaluation of CCTV coverage. Additionally, the plug-in program developed using Revit API, (i.e. CCTV setup advisor) is very helpful for processing the repetitive task of setting up the values of Revit camera parameters. Finally, in the case study of an MRT station, the developed approach is shown to be effective and can be widely applied to other building spaces under similar conditions.

\section{Additional files}

Additional file 1: The CCTV setup advisor.

Additional file 2: The CCTV coverage in the MRT station.

Competing interests

The authors declare that they have no competing interests.

\section{Authors' contributions}

HTC and SWW together developed the robust visualization approach for evaluating the CCTV coverage under the supervision of SHH. The plug-in API program, called the CCTV setup advisor, was developed by HTC and the case study was conducted by both SWW and HTC. All authors contribute to the writing of the manuscript in the way that HTC and SWW drafted the

manuscript and $\mathrm{SHH}$ reviewed and revised it. All authors read and approved the final manuscript.

\section{Acknowledgements}

The authors would like to thank National Science Council, Taiwan for sponsoring the College Student Research Training Fellowship on this research, Department of Rapid Transit Systems, Taipei City Government for 
supporting all related data, drawings, and materials needed by the research, Sinotech Engineering Consultants, Ltd. for sharing a complete BIM model of an MRT station, and ST Electronics (Taiwan) Ltd. for supporting technical assistance of CCTV systems and its practical experiences. Also, the authors appreciate all the comments and suggestions received when an earlier version of this paper was first presented in the 12th International Conference on Construction Applications of Virtual Reality (CONVR 2012), Taipei, Taiwan (Chen et. al, 2012).

Received: 15 February 2013 Accepted: 1 May 2013

Published: 12 June 2013

\section{References}

Aguado, M, Jacob, E, Matias, J, Conde, C, \& Berbineau, M (2009). Deploying CCTV as an Ethenet service over the WiMAX mobile network in the public transport scenario (Proceedings of IEEE International Conference on Communications Workshops (ICC Workshops 2009), pp. 1-5).

Autodesk, Inc. (2010a). Revit Architecture 2011 user's quide. Autodesk, Inc. http:// images.autodesk.com/adsk/files/revit_architecture_2011_user_guide_en.pdf. Accessed 6 Feb 2013.

Autodesk, Inc. (2010b). Revit 2011 API develper's guide. Autodesk, Inc. http://images. autodesk.com/adsk/files/revit2011sdk0.exe. Accessed 6 Feb 2013.

Azhar, S, Hein, M, \& Sketo, B (2008). Building Information Modeling (BIM): benefits, risks and challenges (Proceedings of the 44th ASC Annual Conference). Auburn, Alabama.

Azhar, S, Nadeem, A, Mok, JYN, \& Leung, BHY (2008). Building Information Modeling (BIM): A new paradigm for visual interactive modeling and simulation for construction projects (Proceedings of the First International Conference on Construction in Developing Countries (ICCIDC-I)). Karachi, Pakistan.

Bigdeli, A, Lovell, BC, Sanderson, C, Shan, T, \& Chen, S (2007). Vision processing in intelligent CCTV for mass transport security (Proceedings of the IEEE Workshop on Signal Processing Applications for Public Security and Forensics, pp. 1-4).

CCTV Advisory Service. (2008). LensCalc - online lens calculator. http://www.cctvinformation.co.uk/lenscalc/. Accessed 6 Feb 2013.

Chen, HT, Wu, SW, \& Hsieh, SH (2012). Studying CCTV coverage in an MRT station using BIM-based VR approach (Proceedings of the 12th International Conference on Construction Applications of Virtual Reality (CONVR 2012), pp. 90-98). Taipei, Taiwan.

Cohen, N, Gattuso, J, \& MacLennan-Brown, K (2009). CCTV Operational requirements manual 2009. St Albans: Home Office Scientific Development Branch. http://nactso-dev.co.uk/system/cms/files/127/files/original/ 28_09_CCTV_OR_Manual2835.pdf. Accessed 6 Feb 2013.

Eastman, C, Liston, K, Sacks, R, \& Teicholz, P (2011). BIM Handbook: A guide to building information modeling for owners, managers, designers, engineers \& contractors (2nd ed.). New Jersey: John Wiley \& Sons.

Harris, C, Jones, P, Hillier, D, Turner, D (1998). CCTV surveillance systems in town and city centre management. Property Management, 16(3), 160-165.

Lee, LK, Zachariah, M, \& Everett, P (1995). CCTV camera site selection: a field experience (Proceedings of the Vehicle Navigation and Information Systems Conference (VNIS), pp. 441-446). Washington, D.C.

March Networks News. (2008). Singapore MRT moves ahead on CCTV system expansion. March Networks. http://www.marchnetworks.com/Documents/ Singapore_MRT_Moves_Ahead_on_CCTV_System_Expansion. Accessed 6 Feb 2013.

Sah, V, \& Cory, C (2008). Building Information Modeling: An academic perspective (Proceedings of the 2008 IAJC-IJME International Conference). Nashville, Tennessee.

Sanderson, C, Bigdeli, A, Shan, T, Chen, S, Berglund, E, \& Lovell, BC (2007). Intelligent CCTV for mass transport security: challenges and opportunities for video and face processing. Electronic Letters on Computer Vision and Image Analysis, 6(3), 30-41.

Sun, DS (2005). Closed Circuit Television System (CCTV System) of Taipei Metro. Taipei MRT Newsletter, No. 211. http://www2.dorts.gov.tw/news/newsletter/ ns211/rp211_06.htm. Accessed 6 Feb 2013.

Teague, C, Green, L, \& Leith, D (2010). Watching me watching you: the use of CCTV to support safer work places for public transport transit officers (Proceedings of Australian and New Zealand Communication Association Conference 2010). Canberra, Australia: Australian and New Zealand Communication Association.
Wang, J, Li, J, Chen, X, \& Lv, Z (2010). Developing indoor air quality through healthcare and sustainable parametric method (Proceedings of the 4th International Conference on Bioinformatics and Biomedical Engineering (iCBBE 2010), pp. 1-4). Chengdu, China: IEEE.

Yan, W, Culp, C, \& Graf, R (2011). Integrating BIM and gaming for real-time interactive architectural visualization. Automation in Construction, 20(4), 446-458.

doi:10.1186/2213-7459-1-5

Cite this article as: Chen et al:: Visualization of CCTV coverage in public building space using BIM technology. Visualization in Engineering 2013

$1: 5$.

\section{Submit your manuscript to a SpringerOpen ${ }^{\odot}$ journal and benefit from:}

- Convenient online submission

- Rigorous peer review

- Immediate publication on acceptance

- Open access: articles freely available online

- High visibility within the field

- Retaining the copyright to your article

Submit your next manuscript at $>$ springeropen.com 